\title{
Fractional variational iteration method for solving time-fractional Newell-Whitehead-Segel equation
}

https://doi.org/10.1515/nleng-2018-0001

Received January 4, 2018; revised April 7, 2018; accepted May 5, 2018.

Abstract: In this paper, Newell-Whitehead-Segel equations of fractional order are solved by fractional variational iteration method. Convergence analysis and numerical examples are presented to show the efficiency of the proposed numerical method. Plotted graph demonstrate the mightiness and accurateness of the proposed technique.

Keywords: Fractional variation iteration method; NewellWhitehead-Segel equation of fractional order; Lagrangian multiplier, Caputo fractional derivative.

\section{Introduction}

Nonlinear phenomena are always visible in the study of applied Mathematics, Physics, Chemistry and many related fields of science and engineering. Solving nonlinear system is a herculean task in mathematical analysis and applications. In daily life, we come across many real life models of mathematics for solution of nonlinear fractional differential equation.

The advantage of using fractional models of differential equations in physical models is their non-local property. Fractional order derivative is non-local while integer order derivative is local in nature. It shows that the upcoming state of physical system is also dependent on all of its historical states in addition to its present state. Hence the fractional models are more realistic and fractional derivatives are often used to model problems in acoustics, fluid mechanics, diffusion, electromagnetism, signal processing, biology, finance and some more pro-

\footnotetext{
Amit Prakash, Department of Mathematics, National Institute of Technology, Kurukshetra-136119, India, E-mail: amitmath@nitkkr.ac.in, amitmath0185@gmail.com *Corresponding Author: Manish Goyal, Department of Mathematics, IAH, GLA University, Mathura-281406, India, E-mail: manish.goyal@gla.ac.in,manish101010@gmail.com

Shivangi Gupta, Department of Mathematics, IAH, GLA University, Mathura-281406, India, E-mail: gupta.shivangi151@gmail.com
}

cesses [1-7]. Recently, various methods have been proposed to solve nonlinear fractional differential equations such as Adomian decomposition method [8, 9], differential transform method [10], homotopy perturbation method [11], variational iteration method [12, 13], homotopy analysis method [14-16], homotopy analysis transform method [17-20], homotopy analysis Sumudu transform method [21], homotopy perturbation transform method [22], fractional variational iteration method [2326], q-homotopy analysis transform method $[27,28]$, fractional iteration method $[29,30]$ etc.

Newell-Whitehead-Segel equation is the well-known amplitude equation which also describes the dynamical behavior near the bifurcation point of the Rayleigh-Benard convection of binary fluid mixtures. Rayleigh-Benard convection is a type of natural convection arising in a plane, horizontal layer of fluid heated from below, in which the fluid develops a regular pattern of convection cells known as Bernard cells. When the heating is ample high, convective motion of the fluid develop spontaneously then the hot fluid moves upwards and the cold fluid moves downwards. Rayleigh-Benard convection is one of the most commonly studied convection phenomena because of its analytical and experimental accessibility. The convection patterns are the most carefully examined examples of selforganizing nonlinear systems. Buoyancy and gravity are responsible for the appearance of convection cells. The initial movement is the upwelling of warmer liquid from the heated bottom layer. There are two types of patterns that are noticed normally. First is the roll pattern in which the fluid stream lines form cylinders. These cylinders may be bent and they may form spirals or target-like patterns. Second pattern is the hexagonal one in which the liquid flow is divided into honeycomb cells. For some fluids, the motion is downwards in the center of each cell and upwards on the border between the cells while for other fluids, the motion is in the opposite direction. The same patterns, stripes and hexagons appear in completely different physical systems and on different spatial scales. For example, stripe patterns are observed in human fingerprints, on Zebra's skin and in the visual cortex. Hexagonal patterns result from the propagation of laser beams through a nonlinear medium and in systems with chemical reaction and diffu- 
sion species. Also, the interaction of the diffusion term affect with the effect of nonlinear reaction term is modeled by it.

The Newell-Whitehead-Segel equation [31, 32] of integer order is

$$
\frac{\partial U}{\partial t}=k \frac{\partial^{2} U}{\partial x^{2}}+a U-b U^{q},
$$

where the constants $a, b, k \in R$, with $q$ being a positive integer and $k>0, U(x, t)$ is a function of temporal variable $\mathrm{t}$ and spatial variable $x$, where $t \geq 0, x \in R$. Here $\mathrm{U}$ can be considered as the nonlinear temperature distribution in a thin as well as infinitely long rod. It may also be seen as the fluid flow velocity in a pipe of infinite length having small diameter. The derivative on the L.H.S. of Eq. (1), $\frac{\partial U}{\partial t}$ denotes the partial rate of change in U w.r.t. temporal variable $t$ at a set position. The derivative on the R.H.S. of Eq. (1), $\frac{\partial^{2} U}{\partial x^{2}}$ shows the partial rate of change in U w.r.t. $x$ at a fixed time. Effect of source is shown by the term $a U-b U^{q}$ on the R.H.S. of Eq. (1).

The classical Newell-Whitehead-Segel equations have been studied by Laplace Adomian decomposition method [33], Differential transform method [34], Reduced Differential transform method [35], Adomian decomposition method [36, 37], Homotopy perturbation method [38-40], Iterative method [41], Variational iteration method [42], Finite difference scheme [43], etc.

In this article, we consider the fractional model of Newell-Whitehead-Segel equation of the form

$$
\frac{\partial^{\alpha} U}{\partial t^{\alpha}}=k \frac{\partial^{2} U}{\partial x^{2}}+a U-b U^{q}, 0<\alpha \leqslant 1
$$

where $\alpha$ is a parameter, which describes the order of the time-fractional derivative. The fractional derivative has been taken in Caputo sense. If we take $\alpha=1$, the fractional Newell-Whitehead-Segel Eq. (2) reduces to the classical Newell-Whitehead-Segel Eq. (1).

In fractional differential equations, the general response expression contains a parameter describing the order of the fractional derivative that can be varied to obtain various responses. The time-fractional Newell-WhiteheadSegel equations describe particle motion with memory in time. Space-fractional derivative arises when variations are heavy-tailed and describes particle motion that accounts for variation in the flow field over the entire system. We are focusing more on motion with memory in time. Also, the fraction in time derivative suggests a modulation or weighting of system memory. Therefore, the study of time-fractional Newell-Whitehead-Segel Eq. (2) is very important.

Recently, Kumar and Sharma [44] provided the numerical approximation of Newell-Whitehead-Segel equa- tion of fractional order using homotopy analysis Sumudu transform method (HASTM) and found that homotopy perturbation method (HPM), Adomian decomposition method (ADM) and differential transform method (DTM) are particular cases of the solution obtained by HASTM. The fractional model of Newell-Whitehead-Segel equation has not yet been studied by fractional variational iteration method.

Motivated by the above discussions, in this paper, we propose to study the application of fractional variational iteration method (FVIM) to obtain the numerical solution of time-fractional Newell-Whitehead-Segel Eq. (2) and the results are compared with recently developed technique. This paper is organized in the following manner. Section 1, is introductory. Section 2, presents the brief review of preliminary definitions of Caputo fractional derivative and some other results useful in the study of fractional differential equations. In section 3 , the solution process of FVIM is proposed by taking the problem under consideration. In section 4, we present the sufficient conditions for the convergence of the proposed method and its error estimate. Section 5, presents some numerical test examples on which FVIM is applied to find the approximate solutions and finally in last section 6 , we summarize our results and draw conclusions.

\section{Preliminaries}

Definition 2.1. Consider a real function $\mathrm{h}(\chi), \chi>0$. It is called in space $C_{\zeta}, \zeta \in R$ if $\exists$ a real no. b $(>\zeta)$, s.t. $\mathrm{h}(\chi)=$ $\chi^{b} \mathrm{~h}_{1}(\chi), h_{1} \in C[0, \infty]$. It is clear that $C_{\zeta} \subset C_{y}$ if $y \leq \zeta$. Definition 2.2. Consider a function $\mathrm{h}(\chi), \chi>0$. It is called in space $C_{\zeta}^{m}, m \in N \cup\{0\}$ if $h^{(m)} \epsilon C_{\zeta}$.

Definition 2.3. The left sided Caputo fractional derivative of h, $h \in C_{-1}^{m}, m \in N \cup\{0\}$ is defined as

$D_{t}^{\beta} h(t)=\left\{\begin{array}{l}{\left[I^{m-\beta} h^{(m)}(t)\right], m-1<\beta<m, m \in N,} \\ \frac{d^{m}}{d t^{m}} h(t), \beta=m,\end{array}\right.$

a. $I_{t}^{\zeta} h(x, t)=\frac{1}{\Gamma \zeta} \int_{0}^{t}(t-s)^{\zeta-1} h(x, s) d s ; \zeta, t>0$

b. $D_{\tau}^{v} V(x, \tau)=I_{\tau}^{m-v} \frac{\partial^{m} V(x, \tau)}{\partial \tau^{m}}, m-1<v \leq m$,

c. $D_{t}^{\zeta} I_{t}^{\zeta} h(t)=h(t), m-1<\zeta \leq m, m \in N$,

d. $I_{t}^{\zeta} D_{t}^{\zeta} h(t)=h(t)-\sum_{1}^{m-1} h^{k}(0+) \frac{t^{k}}{k !}, m-1<\zeta \leq$

$m, m \in N$,

e. $I^{v} t^{\zeta}=\frac{\Gamma(\zeta+1)}{\Gamma(v+\zeta+1)} t^{v+\zeta}$.

Definition 2.4. Mittag-Leffler function is demarcated by the given series representation valid in entire complex 
plane:

$$
E_{\zeta}(z)=\sum_{m=0}^{\infty} \frac{z^{m}}{\Gamma(1+\zeta m)}, \zeta>0, z \in C .
$$

\section{Proposed FVIM for the time-fractional Newell-Whitehead-Segel equation}

To illustrate the process of solution by FVIM, we consider the time-fractional Newell-Whitehead-Segel equation

$$
\frac{\partial^{\alpha} U}{\partial t^{\alpha}}=k \frac{\partial^{2} U}{\partial x^{2}}+a U-b U^{q}, 0<\alpha \leqslant 1 .
$$

By FVIM, correction functional is formed as

$$
\begin{aligned}
U_{n+1}(x, t)= & U_{n}(x, t)+\lambda \int_{0}^{t}\left(\frac{\partial^{\alpha} U_{n}(x, \tau)}{\partial \tau^{\alpha}}-k \frac{\partial^{2} \tilde{U}_{n}(x, \tau)}{\partial x^{2}}\right. \\
& \left.-a \tilde{U}_{n}(x, \tau)+b \tilde{U}_{n}(x, \tau)^{q}\right)(d \tau)^{\alpha},
\end{aligned}
$$

where $\lambda$ is a Lagrangian multiplier.

Now, by variational theory, $\lambda$ must satisfy $\left.\frac{d^{\alpha} \lambda}{d \tau^{\alpha}}\right|_{\tau=t}=0$ and

$$
1+\left.\lambda\right|_{\tau=t}=0 \text {. }
$$

Consequently, we obtain $\lambda=-1$ and hence, from equation (3), we get

$$
\begin{aligned}
U_{n+1}(x, t)= & U_{n}(x, t)-\int_{0}^{t}\left(\frac{\partial^{\alpha} U_{n}(x, \tau)}{\partial \tau^{\alpha}}-k \frac{\partial^{2} U_{n}(x, \tau)}{\partial x^{2}}\right. \\
& \left.-a U_{n}(x, \tau)+b U_{n}(x, \tau)^{q}\right)(d \tau)^{\alpha} .
\end{aligned}
$$

Now from Eq. (4), we can obtain successive approximations $U_{n}(x, t), n \geq 0$. The function $U_{n}$ is restricted variation which means $\delta \tilde{U}_{n}=0$. In this way, we get sequences $U_{n+1}(x, t), n \geq 0$.

Finally, the exact solution is obtained as $U(x, t)=$ $\lim _{n \rightarrow \infty} U_{n}(x, t)$.

\section{Convergence analysis}

In this section, we focus on the convergence of the proposed fractional variational iteration method applied to equation (2) in section 3. The sufficient conditions for the convergence of the proposed method and its error estimate [46] are presented.
We define the operator B as:

$$
\begin{aligned}
B= & \int_{0}^{t}(-1)\left(\frac{\partial^{\alpha} U_{n}(x, \tau)}{\partial \tau^{\alpha}}-k \frac{\partial^{2} U_{n}(x, \tau)}{\partial x^{2}}\right. \\
& \left.-a U_{n}(x, \tau)+b U_{n}(x, \tau)^{q}\right)(d \tau)^{\alpha},
\end{aligned}
$$

and we define the components $v_{k}, k=0,1,2, \ldots \ldots$ as,

$$
U(x, t)=\lim _{n \rightarrow \infty} U_{n}(x, t)=\sum_{k=0}^{\infty} v_{k} .
$$

Theorem 1 [47]. Let B, defined in (5), be an operator from a Banach space BS to BS. The series solution $U(x, t)=$ $\lim _{n \rightarrow \infty} U_{n}(x, t)=\sum_{k=0}^{\infty} v_{k}$ as defined in (6), converges if $0<\mathrm{p}<1$ exists such that $\left\|B\left[v_{0}+v_{1}+v_{2}+\ldots+v_{k+1}\right]\right\| \leq$ $p\left\|B\left[v_{0}+v_{1}+v_{2}+\ldots+v_{k}\right]\right\|$, (i.e. $\left\|v_{k+1}\right\| \leq p\left\|v_{k}\right\|$ ), $\forall k \in N \bigcup\{0\}$.

Theorem 1 is a special case of the Banach fixed point theorem, which was used in [48] as a sufficient condition to study the convergence of the FVIM for some partial differential equations.

Theorem 2 [47]. If the series solution $U(x, t)=\sum_{k=0}^{\infty} v_{k}$ defined in (6) converges, then it is an exact solution of nonlinear problem (2).

Theorem 3 [47]. We assume that the series solution $\sum_{k=0}^{\infty} v_{k}$ defined in (6) is convergent to the solution $U(x, t)$. If the truncated series $\sum_{k=0}^{j} v_{k}$ is used as an approximation to the solution $U(x, t)$ of problem (2), then the maximum error, $E_{j}(x, t)$, is estimated as:

$$
E_{j}(x, t) \leq \frac{1}{1-p} p^{j+1}\left\|v_{0}\right\|
$$

If for every $i \in N \bigcup\{0\}$, we define the parameters,

$$
\chi_{i}=\left\{\begin{array}{c}
\frac{\left\|v_{i+1}\right\|}{\left\|v_{i}\right\|},\left\|v_{i}\right\| \neq 0 \\
0,\left\|v_{i}\right\|=0
\end{array}\right.
$$

then the series solution $\sum_{k=0}^{\infty} v_{k}$ of problem (2) converges to an exact solution $U(x, t)$, when

$$
0 \leq \chi_{i}<1,
$$

$\forall i \in N \bigcup\{0\}$. Moreover, as stated in theorem 3, the maximum absolute truncation error is estimated as

$$
\left\|U(x, t)-\sum_{k=0}^{\infty} v_{k}\right\| \leq \frac{1}{1-\chi} \chi^{j+1}\left\|v_{0}\right\|,
$$

where $\chi=\max \left\{\chi_{i}, i=0,1,2, \ldots, j\right\}$.

Remark 1 [47]. If the first finite $\chi_{i}^{\prime} s, i=1,2, \ldots, j$, are not less than one and $\chi_{i} \leq 1$ for $i>j$, then, of course, the series solution $\sum_{k=0}^{\infty} v_{k}$ of problem (2) converges to an exact solution. In other words, the first finite terms do not affect the convergence of series solution. In this case, the convergence of FVIM approach depends on $\chi_{i}$, for $i>j$. 


\section{Numerical Experiments}

In this section, we apply the proposed technique FVIM to some test examples.

Example 1. Consider a linear time-fractional NewellWhitehead-Segel equation

$$
D_{t}^{\alpha} U=U_{x x}-2 U, \quad 0<\alpha \leq 1,
$$

with initial condition

$$
U(x, 0)=e^{x} .
$$

When $\alpha=1$, the exact solution of Eqs. (7) - (8) is $U(x, t)=e^{x-t}$.

The initial solution can be taken as $U_{0}(x, t)=e^{x}$, then

$$
\begin{aligned}
U_{1}(x, t) & =U_{0}-\int_{0}^{t}\left(\frac{\partial^{\alpha} U_{0}}{\partial \tau^{\alpha}}-\frac{\partial^{2} U_{0}}{\partial x^{2}}+2 U_{0}\right)(d \tau)^{\alpha}, \\
& =e^{x}\left(1-\frac{t^{\alpha}}{\Gamma(1+\alpha)}\right), \\
U_{2}(x, t) & =U_{1}-\int_{0}^{t}\left(\frac{\partial^{\alpha} U_{1}}{\partial \tau^{\alpha}}-\frac{\partial^{2} U_{1}}{\partial x^{2}}+2 U_{1}\right)(d \tau)^{\alpha}, \\
& =e^{x}\left(1-\frac{t^{\alpha}}{\Gamma(1+\alpha)}+\frac{t^{2 \alpha}}{\Gamma(1+2 \alpha)}\right), \\
U_{3}(x, t) & =e^{x}\left(1-\frac{t^{\alpha}}{\Gamma(1+\alpha)}+\frac{t^{2 \alpha}}{\Gamma(1+2 \alpha)}-\frac{t^{3 \alpha}}{\Gamma(1+3 \alpha)}\right) .
\end{aligned}
$$

Continuing in this way, the next iterations can be computed by using Mathematica software.

Finally, the solution is found as,

$$
U(x, t)=\lim _{n \rightarrow \infty} U_{n}(x, t)=e^{x} E_{\alpha}\left(-t^{\alpha}\right) .
$$

In view of (5) and (6), the iteration formula for problem (7) can be constructed as,

$$
\begin{aligned}
& v_{0}=e^{x}, \\
& v_{1}=-e^{x} \frac{t^{\alpha}}{\Gamma(1+\alpha)}, \\
& v_{2}=e^{x} \frac{t^{2 \alpha}}{\Gamma(1+2 \alpha)}, \\
& v_{3}=-e^{x} \frac{t^{3 \alpha}}{\Gamma(1+3 \alpha)}, \\
& \vdots \\
& v_{k}=(-1)^{k} e^{x} \frac{t^{k \alpha}}{\Gamma(1+k \alpha)} .
\end{aligned}
$$

Clearly, we can conclude that the obtained solution, $\quad \sum_{k=0}^{\infty} v_{k}$ converges to the exact solution $U(x, t)=e^{x} E_{\alpha}\left(-t^{\alpha}\right)$, where $E_{\alpha}(z)$ is the one parameter Mittag-Leffler function defined in [2]. have,

In addition, by computing $\chi_{i}^{\prime} s$ for this problem, we

$$
\chi_{i}=\frac{\left\|v_{i+1}\right\|}{\left\|v_{i}\right\|}=\left\|t^{\alpha} \frac{\Gamma(1+i \alpha)}{\Gamma(1+(i+1) \alpha)}\right\|<1,
$$

when for example, $i>1$ and $0<\alpha \leq 1$. This confirms that the variational approach for the problem (7)-(8) gives the positive and bounded solution, which converges to the exact solution.

Remark 2 [47]. The above test problem is considered when $0<\mathrm{t} \leq 1$ in order to discuss the condition of convergence. Of course, we can length the interval and examine the condition of convergence after neglecting the first few terms of series solution. For example, if we consider the timefractional Newell-Whitehead-Segel equation (2) when $0<$ $t \leq a$ and $\alpha=1$, where $\mathrm{a}>0$, then $\chi_{i}=\left\|t \frac{\Gamma(1+i)}{\Gamma(1+(i+1))}\right\|=\left\|t \frac{i !}{(i+1) !}\right\| \leq \frac{a}{i+1}<1$, for $i>a$.

Therefore, the series solution $\sum_{k=0}^{\infty} v_{k}$ is positive and bounded, which converges to the exact solution for every a $>0$.

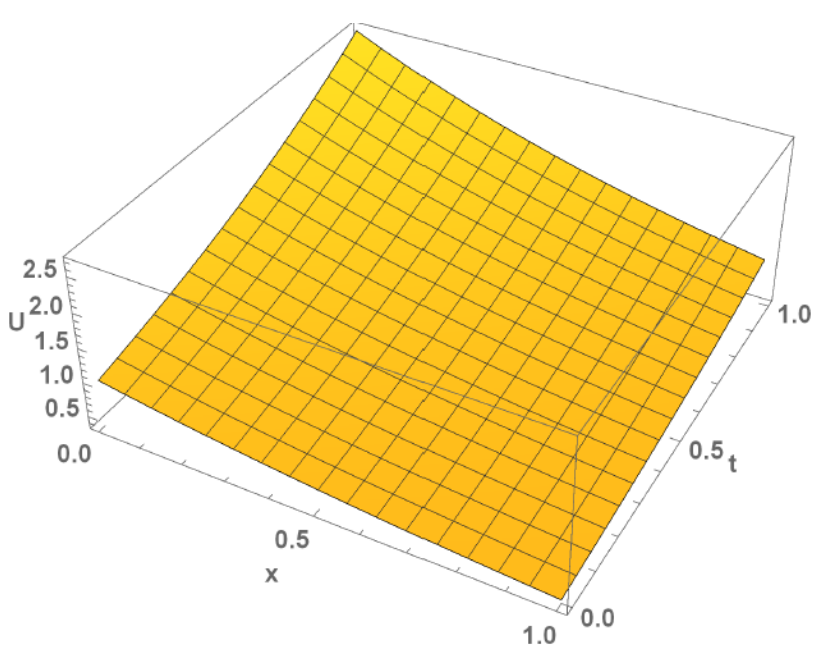

Fig. 1: Behavior of exact solution $U(x, t)$ w.r.t. $\mathrm{x}$ and $\mathrm{t}$ when $\alpha=1$ for Eqs. (7)-(8).

Abbaoui and Cherruault [45] have proved the convergence of this type of series. It is observed from the results that FVIM works efficiently for this problem, though lower order approximate solution $U_{2}(x, t)$ is taken. However, if we take $\alpha=1$, we get the solution of classical NewellWhitehead-Segel equation as

$$
U(x, t)=e^{x}\left[1-\frac{t}{1 !}+\frac{t^{2}}{2 !}+\ldots\right],
$$

which converges very fast to the exact solution

$$
U(x, t)=e^{x-t} .
$$

It is the same solution as obtained by RDTM [35], ADM [36], HPM [39] and HASTM [44]. The numerical results obtained by using FVIM and exact solution are depicted through figs. 1-7. Numerical simulations are carried 


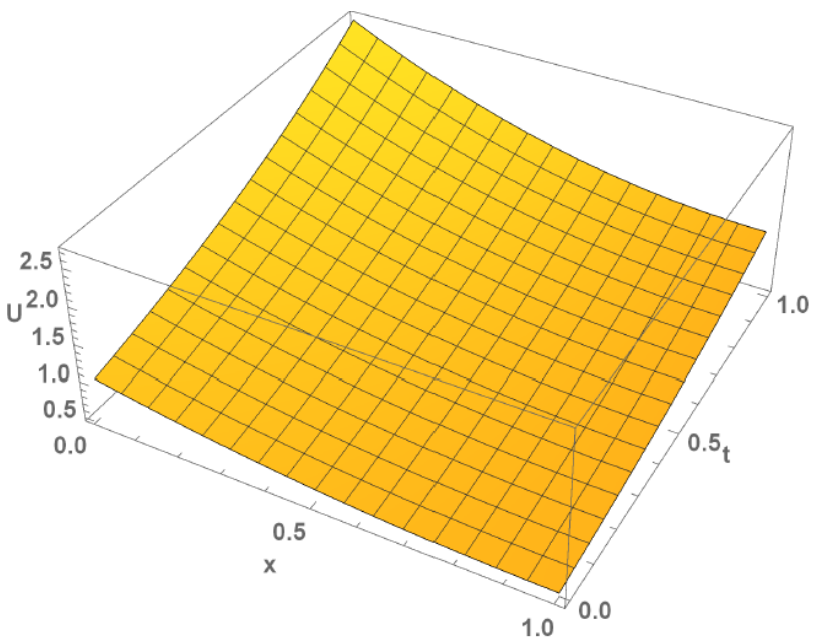

Fig. 2: Behavior of second term approximate solution $U(x, t)$ w.r.t. $\mathrm{x}$ and t, when $\alpha=1$ for Eqs. (7)-(8).

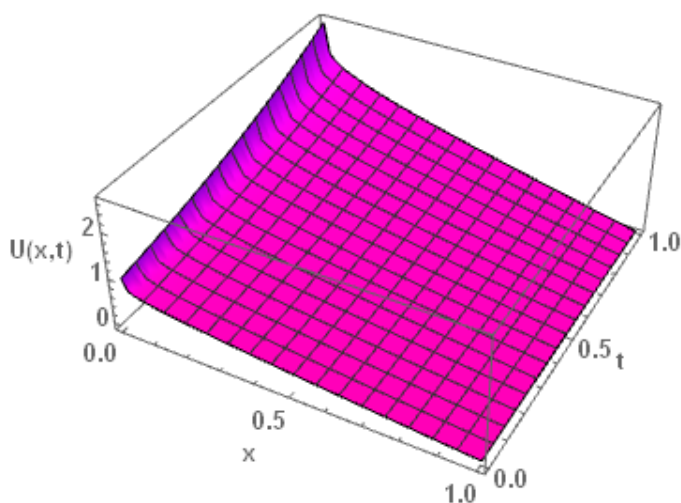

Fig. 3: Behavior of second term approximate solution $U(x, t)$ w.r.t. $\mathrm{x}$ and t, when $\alpha=0.3$ for Eqs. (7)-(8).

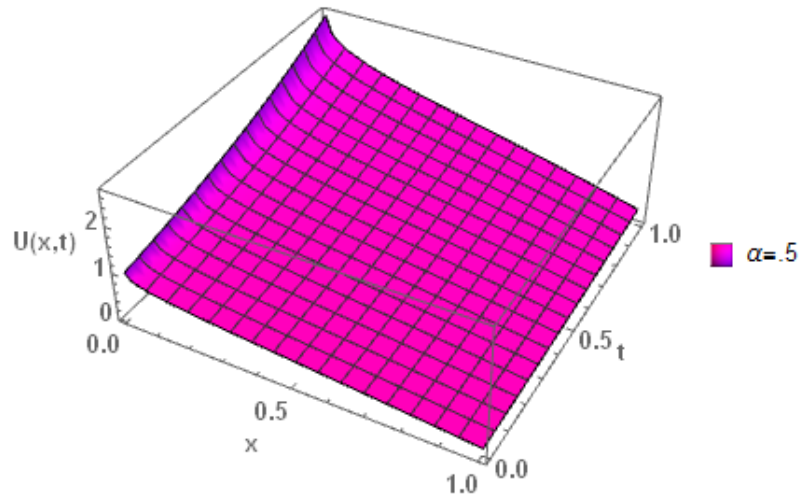

Fig. 4: Behavior of second term approximate solution $U(x, t)$ w.r.t. $\mathrm{x}$ and t, when $\alpha=0.5$ for Eqs. (7)-(8).

out for $U(x, t)$ at the distinct fractional Brownian motions given by $\alpha=0.3,0.5,0.7$ as shown in figs. 3-5 and the standard motion $\alpha=1$ as shown in fig. 2. It is observed

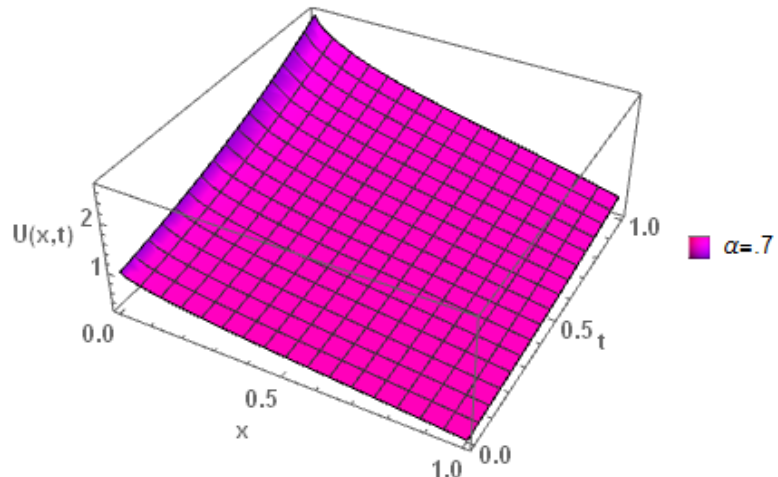

Fig. 5: Behavior of second term approximate solution $U(x, t)$ w.r.t. $\mathrm{x}$ and t, when $\alpha=0.7$ for Eqs. (7)-(8).

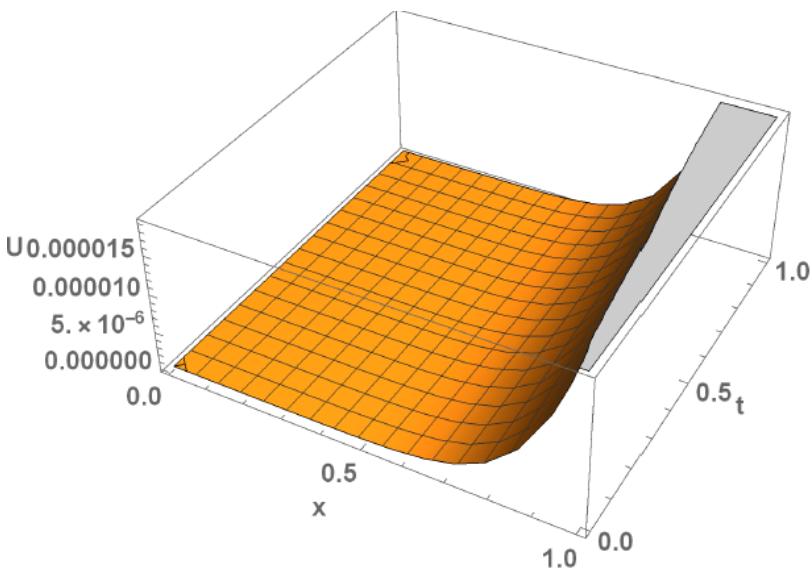

Fig. 6: Absolute error $E_{8}(U)=\mid$ Exact - Approximate value $\mid$ for Eqs. (7) $-(8)$.

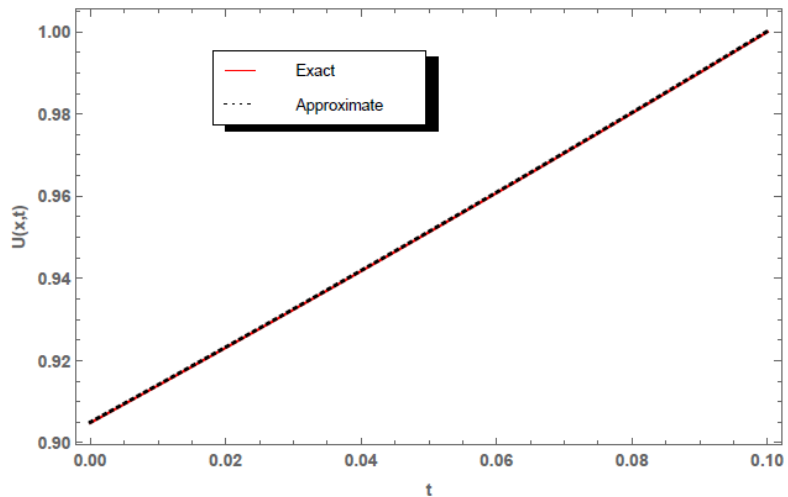

Fig. 7: Comparison between exact and approximate solution when $\alpha=1$ for Eqs. (7) - (8).

from figs. 1-2 that the approximate solution obtained by FVIM is almost identical with exact solution at $\alpha=1$ for different values of $x$ and $t$. From fig. 7, it is found that exact and approximate solutions are in complete agreement at $\alpha=1$. It is also to be noted that only eight terms of the 
series solution are considered for absolute error in fig. 6 . Hence the accuracy of FVIM can be enhanced by increasing the number of iterations.

Example 2. Consider the nonlinear time-fractional Newell-Whitehead-Segel equation

$$
D_{t}^{\alpha} U=U_{x x}+2 U-3 U^{2}, \quad 0<\alpha \leq 1,
$$

with the initial condition

$$
U(x, 0)=\lambda .
$$

When $\alpha=1$, the exact solution of Eqs. (13)-(14) is $U(x, t)=$ $\frac{\frac{-2}{3} \lambda e^{2 t}}{\frac{-2}{3}+\lambda-\lambda e^{2 t}}$.

The initial solution can be taken as $U_{0}(x, t)=\lambda$, then

$$
\begin{aligned}
U_{1}(x, t)= & U_{0}-\int_{0}^{t}\left[\frac{\partial^{\alpha} U_{0}}{\partial \tau^{\alpha}}-\frac{\partial^{2} U_{0}}{\partial x^{2}}-2 U_{0}+3 U_{0}^{2}\right](d \tau)^{\alpha}, \\
= & \lambda+\left(2 \lambda-3 \lambda^{2}\right) \frac{t^{\alpha}}{\Gamma(1+\alpha)}, \\
U_{2}(x, t)= & U_{1}-\int_{0}^{t}\left[\frac{\partial^{\alpha} U_{1}}{\partial \tau^{\alpha}}-\frac{\partial^{2} U_{1}}{\partial x^{2}}-2 U_{1}+3 U_{1}^{2}\right](d \tau)^{\alpha}, \\
= & \lambda+\frac{\left(2 \lambda-3 \lambda^{2}\right)}{\Gamma(1+\alpha)} t^{\alpha}+\frac{2(1-3 \lambda)\left(2 \lambda-3 \lambda^{2}\right)}{\Gamma(1+2 \alpha)} t^{2 \alpha} \\
& -\frac{3 \lambda(2-3 \lambda)^{2} \Gamma(1+2 \alpha)}{(\Gamma(1+\alpha))^{2} \Gamma(1+3 \alpha)} t^{3 \alpha} .
\end{aligned}
$$

Proceeding in this manner, the enduring components can also be obtained using Mathematica software. Hence, we find the solution as $U(x, t)=\lim _{n \rightarrow \infty} U_{n}(x, t)$.

Now, by taking $\alpha=1$, we get the solution of classical nonlinear Newell-Whitehead-Segel equation as

$$
\begin{aligned}
U(x, t)= & +\left(2 \lambda-3 \lambda^{2}\right) \frac{t}{1 !}+2(1-3 \lambda)\left(2 \lambda-3 \lambda^{2}\right) \frac{t^{2}}{2 !} \\
& +6 \lambda(2-3 \lambda)^{2} \frac{t^{3}}{3 !}+\ldots,
\end{aligned}
$$

which converge very fast to the exact solution

$$
U(x, t)=\frac{\frac{-2}{3} \lambda e^{2 t}}{\frac{-2}{3}+\lambda-\lambda e^{2 t}} .
$$

It is the same solution as obtained by RDTM [35], ADM [36], HPM [39] and HASTM [44]. The numerical results obtained by using FVIM and exact solution are presented through fig. 8 at the distinct fractional Brownian motions given by $\alpha=0.25,0.5,0.75$ and the standard motion $\alpha=1$ at $\lambda=1$ for different values of $t$. It is observed that for $\alpha=0.25$, as $\mathrm{t}$ increases, $\mathrm{U}$ first decreases but then sharply increases afterwards. For $\alpha=0.5$ also,
$\mathrm{U}$ first decreases and then increases with increasing t. It is interesting to note that $\mathrm{U}$ decreases with increasing $\mathrm{t}$ for values of $\alpha \geq 0.7$ and finally approaches the exact solution at $\alpha=1$. fig. 8 shows that the results obtained with the help of FVIM are approximately same as the exact solution at $\alpha=1$. The numerical results indicate that FVIM works very well, even if lower order approximate solution $U_{2}(x, t)$ is used. However, the accuracy can be improved by using higher order approximations in solution.

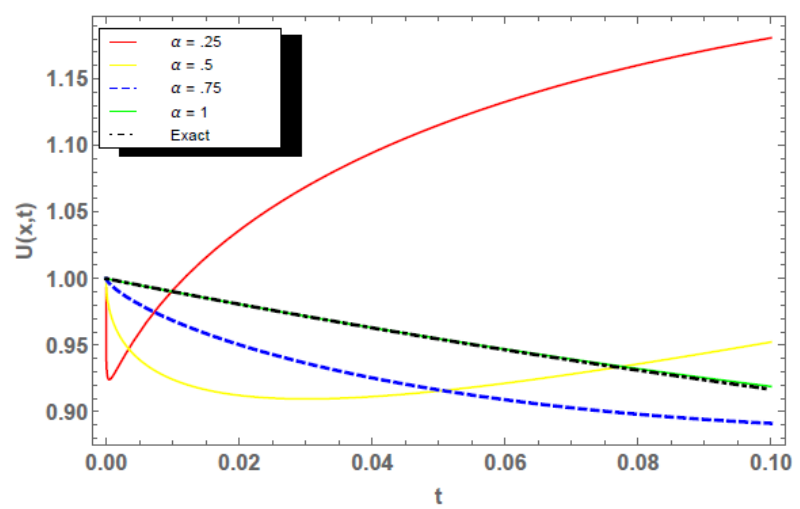

Fig. 8: Plots of $U(x, t)$ vs. $t$ at $\alpha=0.25,0.5,0.75$ and $\alpha=1$ at $\lambda=1$ for exact solution and approximate solution for Eqs. (13)-(14).

\section{Conclusion}

In this article, FVIM is successfully applied to solve fractional model of Newell-Whitehead-Segel equation. It is apparently seen from illustrative example that FVIM is easy to implement, powerful and efficient numerical method to find an approximate solution. It is also to be noted that FVIM is used directly without using linearization, perturbation, Adomian polynomial or any restrictive assumptions. Hence, FVIM is more convenient and also easier than other existing methods.

Acknowledgement: The authors are thankful to the anonymous reviewers for their valuable comments and suggestions to improve the quality of the paper.

\section{References}

[1] R. Hilfer, Applications of fractional Calculus in Physics, World Scientific publishing Company, Singapore-New Jersey-Hong Kong 2000 87-130. 
[2] I. Podlubny, Fractional Differential Equations, Academic Press, New York 1999.

[3] M.A. E. Herzallah, A.M.A. El-Sayed, D. Baleanu, On the fractional-order diffusion-wave process, Rom. J. Phys. 55(34) (2010) 274-284.

[4] M. Caputo, Elasticita Dissipazione, Zani-Chelli, Bologna 1969.

[5] K. S. Miller, B. Ross, An Introduction to the fractional calculus and fractional differential equations, Wiley, New York 1993.

[6] A. A. Kilbas, H. M. Srivastava, J. I. Trujillo, Theory and applications of fractional differential equations, Elsevier, Amsterdam 2006.

[7] Z. Odibat, S. Momani, Numerical methods for non-linear partial differential equations of fractional order, Appl. Math. Model. 32 (2008) 28-39.

[8] A. M. Wazwaz, A new algorithm for calculating Adomian polynomials for nonlinear operators, Appl. Math. Comput. 111 (2000) 33-51.

[9] Z. Hammouch, T. Mekkaoui, Approximate analytical and Numerical solutions to fractional KPP-like equations, Gen. Math. Notes 14 (2) (2013) 1-9.

[10] A. Arikoglu, I. Ozkol, Solution of fractional differential equations by using differential transform method, Chaos, Solitons Fractals 34 (2007) 1473-1481.

[11] J. H. He, Homotopy perturbation technique, Comput. Methods in Appl. Mech. Eng. 178 (1999) 257-262.

[12] J. H. He, Variational iteration method-a kind of non-linear analytical technique: Some examples, Int. J. Nonlinear Mech. 34 (1999) 699-708.

[13] J. H. He, X. H. Wu, Variational iteration method: new development and applications, Comput. Math. Appl. 54 (2007) 881894.

[14] J. Singh, D. Kumar, R. Swroop, S. Kumar, An efficient computational approach for time-fractional Rosenau-Hyman equation, Neural Comput. \& Applic. (2017), doi 10.1007/s00521-0172909-8

[15] H. Jafari, A. Golbabai, S. Seifi, K. Sayevand, Homotopy analysis method for solving multi-term linear and nonlinear diffusion wave equations of fractional order, Comput. Math. Appl. 59 (2010) 1337-1344.

[16] X. Zhang, B. Tang, Y. He, Homotopy analysis method for higher-order fractional integro-differential equations, Comput. Math. Appl. 62 (2011) 3194-3203.

[17] A. Kumar, S. Kumar, A modified analytical approach for fractional discrete $\mathrm{KdV}$ equations arising in particle vibrations, Proc. Natl. Acad. Sci., India, Sect. A Phys. Sci., 88 (2018) 95106.

[18] S. Kumar, A. Kumar, Z. M. Odibat, A nonlinear fractional model to describe the population dynamics of two interacting species, Math. Meth. App. Sci, 40 (11) (2017) 4134-4148.

[19] S. Kumar, A. Kumar, I. K. Argyros, A new analysis for the KellerSegel model of fractional order, Numerical Algorithms 75(1) (2017) 213-228.

[20] S. Kumar, D. Kumar, J. Singh, Fractional modelling arising in unidirectional propagation of long waves in dispersive media, Advances in Nonlinear Analysis, 5(4) (2016) 2013-2033.

[21] S. Rathore, D. Kumar, J. Singh, S. Gupta, Homotopy analysis Sumudu transform method for nonlinear equations, Int. J. Industrial Mathematics 4(4) (2012) 301-314.

[22] A. Prakash, Analytical method for space-fractional telegraph equation by homotopy perturbation transform method, Nonlin- ear Engineering 5(2) (2016) 123-128.

[23] A. Prakash, M. Kumar and K. K. Sharma, Numerical method for solving fractional coupled Burgers equations, Appl. Math. Comput. 260 (2015) 314-320.

[24] A. Prakash, M. Kumar, Numerical method for solving timefractional multi- dimensional diffusion equations, Int. J. Computing Science and Mathematics 8(3) (2017) 257-267.

[25] A. Prakash, M. Kumar, Numerical method for fractional dispersive partial differential equations, Communications in Numerical Analysis 2017 (1) (2017) 1-18.

[26] A. Prakash, M. Kumar, Numerical solution of two dimensional time fractional-order biological population model, Open Physics 14 (1) (2016) 177-186.

[27] A. Prakash, H. Kaur, Numerical solution for fractional model of Fokker-Planck equation by using q-HATM, Chaos, Solitons and Fractals 105 (2017) 99-110.

[28] A. Prakash, H. Kaur, q-homotopy analysis transform method for space and time- fractional KdV-Burgers equation, Nonlinear Sci. Lett. A 9 (1) (2018) 44-61.

[29] T. Mekkaoui, Z. Hammouch, Approximate analytical solutions to the Bagley-Torvik equation by the Fractional iteration method, Annals of the University of Craiova, Mathematics and Computer Science Series 39(2) (2012) 251-256.

[30] Z. Hammouch, T. Mekkaoui, Approximate analytical solution to a time-fractional Zakharov-Kuznetsov equation, International Journal of Physical Research 1(2) (2013) 28-33.

[31] A. Newell and J. Whitehead, Finite bandwidth finite amplitude convection, J. Fluid Mech. 38 (1969) 279 - 303.

[32] M. M. A. Mangoub, A. K. H. Sedeeg, On the solution of NewellWhitehead-Segel equation, Am. J. Math. Comput. Model. 1 (2016) 21-24.

[33] P. Pue-on, Laplace Adomian decomposition method for solving Newell-Whitehead-Segel equation, Appl. Math. Sci. 7 (2013) 6593-6600.

[34] A. Aasaraai, Analytic solution for Newell-Whitehead-Segel equation by differential transform method, Middle-East J. Sci. Res. 10 (2011) 270-273.

[35] A. Saravanan, N. Magesh, A comparison between the reduced differential transform method and the Adomian decomposition method for the Newell-Whitehead-Segel equation, J. Egyptian Math. Soc. 21 (2013) 259-265.

[36] S. A. Manaa, An Approximate solution to the NewellWhitehead-Segel equation by the Adomian decomposition method, Raf. J. Comp. Math. 8 (2011) 171-180.

[37] A. A. Aswhad, A. F. Jaddoa, The approximate solution of Newell-Whitehead-Segel and Fisher equation using the Adomian Decomposition Method, Al-Mustansiriyah J. Sci. 25(4) (2014).

[38] H. K. Jassim, Homotopy perturbation algorithm using Laplace transform for Newell-Whitehead-Segel equation, Int. J. Adv. Appl. Math. Mech. 2 (2015) 8-12.

[39] S. S. Mourazar, M. Soori and A. Nazari-Golsham. On the exact solution of Newell-Whitehead-Segel equation using the homotopy perturbation method, Aust. J. Basic Appl. Sci. 5 (2011) 1400-1411.

[40] M. A. Mahgoub, Homotopy Perturbation Method for Solving Newell-Whitehead-Segel Equation, Adv. Theor. Appl. Math. 11 (2016) 399-406.

[41] J. Patade, S. Bhalekar, Approximate analytical solution of Newell-Whitehead-Segel equation using a new iterative 
method, World J. Model Simul. 11 (2015) 94-103.

[42] A. Prakash, M. Kumar, He's Variational Iteration Method for solution of nonlinear Newell- Whitehead-Segel equation, J. Appl. Anal. Comput. 6(3) (2016) 738-748.

[43] J. E. Macias-Diaz, J. Ruiz-Ramirez, A non-standard symmetrypreserving method to compute bounded solutions of a generalized Newell-Whitehead-Segel equation, Appl. Numer. Math. 61 (2011) 630-640.

[44] D. Kumar, R. P. Sharma, Numerical approximation of NewellWhitehead-Segel equation of fractional order, Nonlinear Engineering 5(2) (2016) 81-86.
[45] K. Abbaoui, Y. Cherruault, New ideas for proving convergence of decomposition methods, Comput. Math. Appl. 29 (7) (1995) 103-108.

[46] M. G. Sakar, H. Ergoren, Alternative variation iteration method for solving the time- fractional Fornberg-Whitham equation, Appl. Math. Model. 39(14) (2015) 3972-3979.

[47] Z. M. Odibat, A study on the convergence of variational iteration method, Math. Comput. Model. 51 (2010) 1181-1192.

[48] M. Tatari, M. Dehghan, On the convergence of He's variational iteration method, J. Comput. Appl. Math. 207 (1) (2008) 121128. 